\title{
Short-course multidrug therapy for leprosy patients in Western Kenya. Preliminary communication
}

\author{
P A OREGE, M OBURA \& J O NYAWALO \\ Kenya Medical Research Institute, Alupe Leprosy and Skin Diseases \\ Research Centre, PO Box 3, Busia, Kenya
}

Accepted for publication 23 June 1986

\begin{abstract}
Summary A prospective study has been undertaken to evaluate how tolerable and effective short-course multidrug chemotherapeutic regimens are, for both multibacillary and paucibacillary cases of leprosy, using WHO-recommended regimens. For paucibacillary cases, we have compared the WHO recommended multidrug regimen and a modified multidrug regimen, consisting of rifampicin $1500 \mathrm{mg}$ at the outset and repeated after 3 months, together with dapsone $100 \mathrm{mg}$ daily unsupervised. The WHO recommended multidrug regimen for multibacillary cases of leprosy is given for 2 years, after which the cases are followed for 3 years. Paucibacillary cases on WHO-recommended and modified MDT will get active therapy for 6 months after which they will be followed for 18 months.

A total of 145 patients were admitted to the study, out of which $37(25 \cdot 5 \%)$ were multibacillary and $108(75 \cdot 5 \%)$ paucibacillary cases. Of the registered cases $93(64.1 \%)$ are females and $52(35.9 \%)$ are males. Within the period of study, a quite high clinical cure rate was noted amongst paucibacillary cases, in many instances from the second month onwards. The default rate was low and no adverse effects associated with the use of either clofazimine or rifampicin were noted. One female patient developed exfoliative dermatitis, thought to be due to dapsone, which completely cleared once the use of this drug had been stopped, and another female patient had hypochromic anaemia, calling for transfusion, also possibly related to dapsone. Contrary to reports from some other centres on continuing activity in paucibacillary cases even after the full course of WHOrecommended therapy, our preliminary experience in Kenya suggests that clinical cure can be achieved in much shorter periods than had hitherto been supposed.
\end{abstract}

\section{Introduction}

In spite of intensive control efforts, leprosy still remains a major public health hazard in Kenya and throughout the world. There are estimated 11 million cases of leprosy in the world, ${ }^{1}$ and approximately 1.4 billion people are still at risk of contracting leprosy. ${ }^{2}$ In Kenya there are approximately 50,000 cases out of which 11,000 are registered, ${ }^{3}$ and approximately 11 million people are still at risk of 
contracting the disease. At present the only effective method available for controlling this disease is effective chemotherapy, i.e. early case detection and effective treatment of the detected cases. Dapsone as a monotherapy, has been used in the treatment of leprosy since it was first used in 1949 by Dr Cochrane in India, ${ }^{4}$ and in Kenya it has been used for treatment of leprosy cases since $1952 .{ }^{5}$ Although sulphone resistance has been suspected since 1950s, the resistance of Mycohacterium leprae to dapsone per se was first proved in mouse foot pad in $1964 .{ }^{6}$ Subsequently dapsone resistance has been proved in more than 25 countries of the world, ${ }^{7}$ Kenya included. ${ }^{5}$ Hence in recent years the limitation to dapsone monotherapy has become increasingly obvious. ${ }^{7}$ In addition to the threat of resistance, dapsone has slow effect, which inevitably results in slow clinical improvement, long-term treatment, and relapse.

Many patients therefore lose faith in dapsone resulting in a high drop out rate and irregularity. ${ }^{8}$ To overcome these problems, $\mathrm{WHO}^{9}$ recommended short-term therapy with rifampicin to all patients. The recommended regimen for multibacillary cases of leprosy was rifampicin $600 \mathrm{mg}$ monthly supervised, clofazimine $300 \mathrm{mg}$ monthly supervised, dapsone $100 \mathrm{mg}$ daily unsupervised and clofazimine $50 \mathrm{mg}$ daily unsupervised. The duration of treatment being a minimum of 2 years. The regimen for paucibacillary cases of leprosy was rifampicin $600 \mathrm{mg}$ once a month supervised with dapsone $100 \mathrm{mg}$ daily unsupervised, for a period of 6 months. ${ }^{9}$ Alupe Leprosy and Skin Diseases Research Centre decided to conduct pilot trials of short-course multidrug regimens as recommended by WHO to find out the effectiveness, efficacy, acceptability, toxicity and any side-effects or unacceptable reactions. In addition to the WHO-recommended regimens, we were also interested in developing an alternative regimen that would be slightly cheaper and easier to administer than the WHO regimen for paucibacillary cases. Therefore we decided to compare the WHO recommended regimen with a modified regimen of rifampicin $1500 \mathrm{mg}$ lst month (supervised) and then to be repeated after 3 months (supervised) plus dapsone $100 \mathrm{mg}$ self-administered daily for 6 months. The aim of this was to determine if the latter regimen could be comparable to WHO regimen in terms of efficacy and eventual effectiveness.

\section{Materials and methods}

A pilot trial was done on both multibacillary and paucibacillary cases of leprosy. The criteria for admission into trial for multibacillary cases of leprosy were as follows:

All newly diagnosed cases who had a positive skin smear with bacteriological index (BI) of $2+$ and above and who agreed to participate in the trial.

2 Multibacillary leprosy cases who had been on dapsone monotherapy for at most 1 year, and still had active clinical signs and symptoms, still had a positive 
skin smear with a bacteriological index of $2+$ and who agreed to participate in the trial.

The criteria for admission of paucibacillary cases were as follows:

1 All newly diagnosed cases who had bacteriological index (BI) of less than $2+$. 2 All paucibacillary cases who had been on dapsone monotherapy for at most 1 year, still had active signs and symptoms and who had bacteriological index of 0 $1+$ in skin smear.

All new patients who were eligible and who were diagnosed in peripheral health clinics and Alupe Research Clinic were included into study.

Paucibacillary cases who agreed to participate in the trial were allocated to either WHO-recommended or MDT-modified regimen randomly, but special considerations were taken by the principal investigator on the age, and also the distance where the patient was coming from in case of Alupe clinic.

The patients considered for the trial and who agreed to participate were clinically examined, undressed in good light, and the lesions marked on gridsystem diagrams. Enlargement of peripheral nerves were noted and tentative diagnosis was made whether a case was a multibacillary or paucibacillary. Skin smears were taken from six sites including at least one active site. Lepromin test was done by injecting either $0.1 \mathrm{ml}$ standard Dharmendra antigen or $0.1 \mathrm{ml}$ of armadillo derived leprosin $\mathrm{A}$ intradermally on the flexor surface of the left lower arm. Urine was taken for simple albumen and sugar tests, and for dapsone analysis. Blood was taken for a full hemogram, Khan test and liver function tests. Stool was taken for examination of ova and cyst.

Voluntary muscle testing and sensory testing (VMT/ST) were also performed on the patients. After full examination and collection of specimens, the patients were given rifampicin which they swallowed on the spot. For multibacillary cases the patients were also given clofazimine $300 \mathrm{mg}$. The patients were then given dapsone tablets to take home. Sufficient dapsone (and clofazimine in case of multibacillary cases) was given so that the patient would swallow the last tablet on the eve of next clinic day. The patients were then given appointments for the next clinic day. The appointment dates were marked on patients appointment cards.

During the next clinic visit the patients were initially asked about the sideeffects of the drugs, after which they were clinically re-examined to find out if there were any changes of the skin lesions. After clinical examination, urine was collected from each patient for routine analysis and dapsone compliance tests.

The patients were then given their supervised treatment and dapsone to be self-administered at home. At the third month of review the patients had repeat skin smears, hematological and liver function tests. These were again performed at the end of 6 months. Paucibacillary patients who had finished their 6 months treatment were changed to placebo, while multibacillary cases will be changed to placebo after 2 years of review (intensive phase treatment). 


\section{Results}

A total of 145 patients were registered on treatment out of which $37(25 \cdot 3 \%)$ were multibacillary and $108(74.5 \%)$ were paucibacillary. Of the registered cases 93 $(64.1 \%)$ were females and $52(35.9 \%)$ were males. Male to female ratio being 1:2. The patients were drawn from 15 treatment centres, those from Alupe research clinic being $26 \%$ of the total. The monthly admission rate was nearly uniform, with an average of 22 cases (multibacillary and paucibacillary) per month, except for the months of August and September when the admission was low, i.e. 14 and 12 cases respectively.

The youngest person in the study was a 6-year-old male multibacillary case and the oldest person in the study was an 80 -year-old female paucibacillary case. As can be seen from Table 1 the age distribution of the admitted cases shows preponderence in the over 50 age-group (29\%) as compared with 0-9 age group $1.4 \%$. From Table 1 it can also be noted that both male and female adults accounted for $84.1 \%$ of the cases, and that females were the majority in all the age groups. Table 2 shows the distribution of paucibacillary cases admitted per each regimen. So far 49 paucibacillary cases have been admitted on MDT-modified and 52 cases have been admitted on MDT-WHO recommended. Seven cases who were below 15 years of age were allocated to WHO recommended regimen for children. Results of the clinical assessment done on study cases every 4 weeks for paucibacillary cases is shown in Table 3 . Eight cases (7.4\%) attained clinical inactivity at the second month of treatment i.e. between 5-8 weeks. Clinical inactivity here is defined as the repigmentation of the hypopigmented patches i.e. disappearance of the skin signs. Among the 8 patients who attained clinical

Table 1. The age distribution of leprosy cases on multidrug therapy trial in Western Kenya between March 1985 and October 1985 by sex

\begin{tabular}{lccc}
\hline & \multicolumn{3}{c}{ Sex } \\
\cline { 2 - 3 } Age (years) & Male & Female & Total \\
\hline $0-9$ & 1 & 1 & $2(1 \cdot 38 \%)$ \\
$10-19$ & 7 & 14 & $21(14 \cdot 48 \%)$ \\
$20-29$ & 4 & 9 & $13(18 \cdot 97 \%)$ \\
$30-39$ & 13 & 15 & $28(19 \cdot 31 \%)$ \\
$40-49$ & 8 & 16 & $24(16 \cdot 55 \%)$ \\
$50+$ & 19 & 23 & $42(28 \cdot 97 \%)$ \\
Not known & 0 & 15 & $15(10 \cdot 34 \%)$ \\
Total & $52(35 \cdot 86 \%)$ & $93(64 \cdot 14 \%)$ & $145(100 \%)$ \\
& \multicolumn{3}{c}{} \\
\hline
\end{tabular}


Table 2. The distribution of paucibacillary leprosy cases on multidrug therapy trial in Western Kenya between March 1985 and October 1985 according to the type of regimen and sex

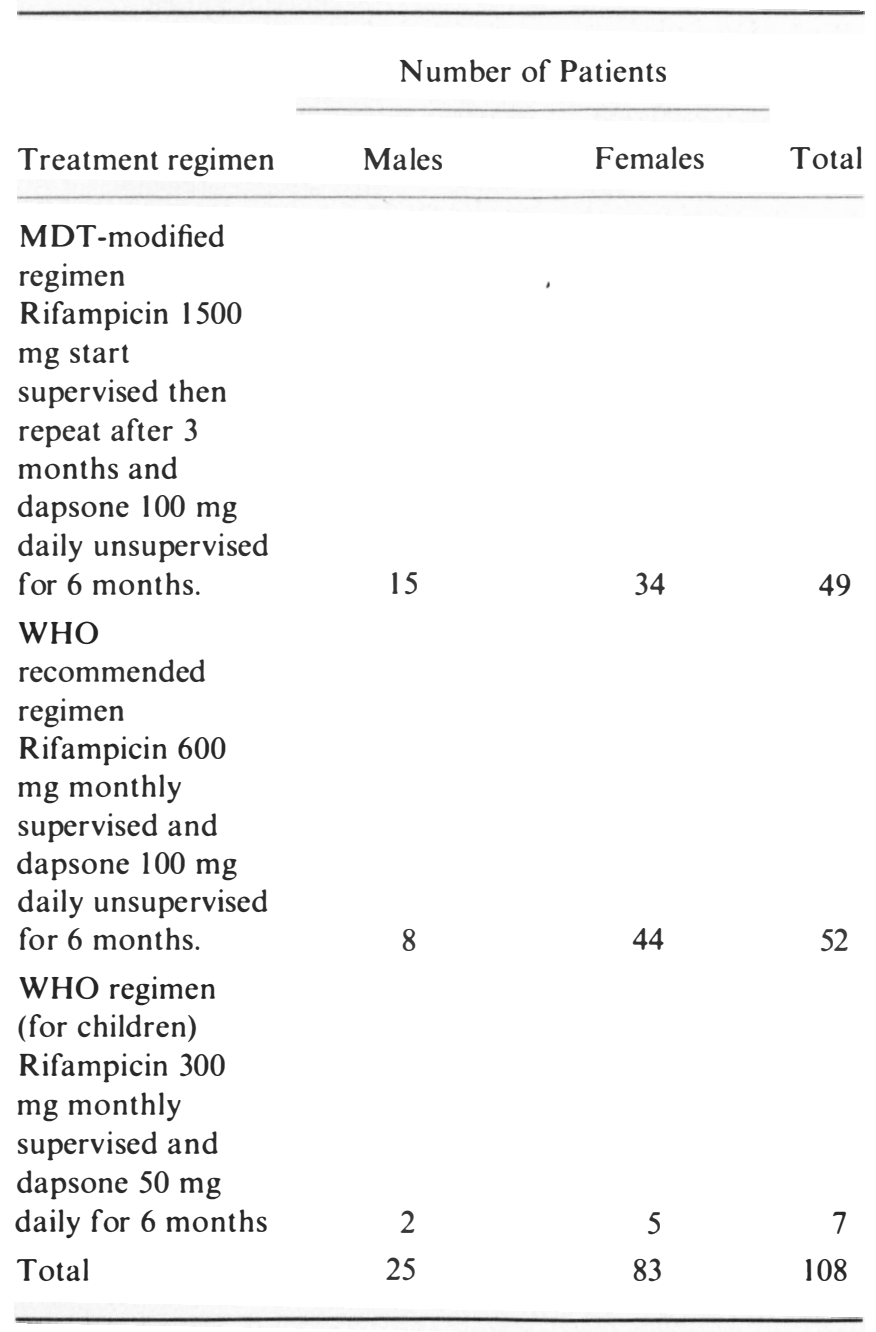

inactivity at the second month of treatment/review, 7 patients were on MDTmodified regimen and 1 patient was on WHO-recommended regimen. Nine $(8.3 \%)$ cases showed clinical inactivity at the third month of treatment/review, i.e. between 9 and 12 weeks. Twenty-one (19.4\%) cases showed clinical inactivity between 13 and 16 weeks. Five (5.1\%) cases showed clinical inactivity between 17 and 20 weeks. Of the total cases $38.9 \%$ were shown to be either resolving or regressing. Some of these latter cases had been admitted into study during the later months before the analysis and are still being followed-up for reviews/ treatment. Eight $(7 \cdot 4 \%)$ cases defaulted from treatment. Among those who 
Table 3. The clinical inactivity (cure) rates by time in weeks of paucibacillary cases of leprosy on multidrug trial studies in Western Kenya between March 1985 and October 1985 by age, sex and the type of regimen, i.e. WHO regimen and MDT-modified regimen

\begin{tabular}{|c|c|c|c|}
\hline \multirow[t]{3}{*}{ Time (weeks) } & $\begin{array}{l}\text { Regimen MDT- } \\
\text { modified Rifampi- } \\
\text { cin } 1500 \mathrm{mg} \text { start } \\
\text { supervised then re- } \\
\text { peat after } 3 \text { months } \\
\text { supervised and dap- } \\
\text { sone } 100 \mathrm{mg} \text { daily } \\
\text { supervised for } 6 \\
\text { months }\end{array}$ & $\begin{array}{l}\text { Regimen-WHO re- } \\
\text { commended } \\
\text { Rifampicin } 600 \mathrm{mg} \\
\text { monthly supervised } \\
+ \text { dapsone } 100 \mathrm{mg} \\
\text { daily unsupervised } \\
\text { for } 6 \text { months or ri- } \\
\text { fampicin } 300 \mathrm{mg} \\
\text { monthly supervi- }\end{array}$ & \multirow[b]{3}{*}{ Total } \\
\hline & & $\begin{array}{l}\text { sed + dapsone } 100 \\
\text { mg daily }\end{array}$ & \\
\hline & No. of leprosy cases & No. of leprosy cases & \\
\hline $0-4$ & 0 & 0 & $0(0 \cdot 0 \%)$ \\
\hline $5-8$ & 7 & 1 & $8(7 \cdot 4 \%)$ \\
\hline $9-12$ & 4 & 5 & $9(8 \cdot 3 \%)$ \\
\hline $13-16$ & 10 & 11 & $21(19 \cdot 4 \%)$ \\
\hline $17-20$ & 2 & 3 & $5(4 \cdot 6 \%)$ \\
\hline $21-24$ & 4 & 4 & $8(7 \cdot 4 \%)$ \\
\hline $25-28$ & 1 & 3 & $4(3 \cdot 7 \%)$ \\
\hline $29-32$ & 1 & 1 & $2(1 \cdot 9 \%)$ \\
\hline $32+$ & 0 & 0 & $0(0 \cdot 0 \%)$ \\
\hline \multicolumn{4}{|l|}{ Regressing } \\
\hline or resolving & 18 & 24 & $42(38.9 \%)$ \\
\hline Total & 47 & 52 & $99(91 \cdot 7 \%)$ \\
\hline
\end{tabular}

* Not included: 1 Neural patient on rifampicin (0.93\%); 8 cases who absconded ( 3 patients on rifampicin $1500 \mathrm{mg}$; and 5 patients on rifampicin $600 \mathrm{mg})(7 \cdot 4 \%)$.

absconded from treatment were 3 cases ( 1 male, 2 females) who were on MDTmodified regimen and 5 cases (all females) who were on WHO-MDT regimen. No side-effects due to either rifampicin or clofazimine (in the case of multibacillary leprosy) were noticed. Only one 14-year-old female case developed exfoliative dermatitis, which we thought was due to dapsone, and which disappeared when dapsone was stopped. Liver function tests have remained within normal limits on all study cases up to date. One female paucibacillary case developed hypochronic anaemia, and had to be transfused while on therapy. However when dapsone was stopped her haemoglobin level started to rise again.

For paucibacillary cases, the level of type 1 reaction has been $9 \cdot 45 \%$ for those 
on MDT-modified regime, and $11.02 \%$ on those MDT-WHO recommended regimen. This indeed has not been greater than the patients on dapsone monotherapy. Five paucibacillary cases $(4.6 \%)$ had positive skin smears on admission, they all became negative in the third month, and all five had negative skin smears at the time of discharge from treatment.

Five per cent of cases who were on MDT modified developed mild type 1 reaction and were treated on outpatient basis. Of MDT-WHO cases $5 \cdot 2 \%$ also developed mild type 1 reaction, which did not require admission into hospital. Among those who developed type 1 reaction $2 \%$ had positive skin smears initially, i.e. before treatment.

So far we have discharged 32 paucibacillary cases from treatment, and these are being followed-up for relapse on placebo. The cases who have been discharged from intensive phase treatment are still reviewed every month together with the patients who are still on active therapy.

\section{Discussion}

One hundred and forty-five patients have been admitted to short-course multidrug chemotherapy trials in the last 8 months. It is still too early to comment on clinical progress of multibacillary cases of leprosy. Hence we can only comment on paucibacillary cases of leprosy, some of whom have already completed their required 6 months treatment. It is also still too early to comment on the effectiveness of multidrug therapy on paucibacillary cases of leprosy, hence we can only concentrate on efficacy, tolerability and acceptability.

Preliminary results indicate that the efficacy of multidrug therapy treatment of paucibacillary cases of leprosy may be high. Clinical inactivity occurred in $7 \cdot 4 \%$ (8) of the cases in the 2 nd month of review. Among these 7 cases $(87.5 \%)$ were on a higher start dose of rifampicin, i.e. rifampicin $1500 \mathrm{mg}$ start. This therefore shows that paucibacillary leprosy cases can respond to multidrug therapy much faster if the initial dose of rifampicin is increased to a maximum tolerable level. Studies done in Trinidad and Tobago, ${ }^{10}$ Guyana,,${ }^{11}$ India,${ }^{8}$ show that multidrug therapy can be effective in treatment of paucibacillary cases of leprosy.

Keeler $R F^{10}$ found that clinically, lesions respond more rapidly on patients on multidrug therapy in which rifampicin is included than on monotherapy or dual therapy where rifampicin is absent. He also noticed that clinical improvement occurred around 2 weeks in most of the patients. Revankar $C^{8}{ }^{8}$ conducting his study in Greater Bombay area found that the addition of rifampicin on pulse therapy basis increased compliance rate significantly $(91 \%)$ as compared to dapsone attendance rate $(64 \%)$. He also found that the addition of rifampicin hastened the clinical regression of single lesion tuberculoid patients. These findings concur with ours, in the sense that we also noticed rapid clinical 
regression on patients who had less than 3 lesions and the regression was much faster on patients who were taking higher dosage of rifampicin as an outset dose.

In conclusion, though it is too early yet to give a definitive view on shortcourse multidrug regimen on paucibacillary cases of leprosy, it can be said that the addition of rifampicin to dapsone monotherapy does, indeed, hasten the cure of these patients and also reduce the default rate. The default rate of our study patients was $7 \cdot 4 \%$ as compared with more than $50 \%$ of the patients on dapsone monotherapy.

Once paucibacillary patients are cured and discharged from treatment, more time can be devoted to multibacillary cases, making leprosy control more effective than it could have been on dapsone monotherapy. The feared complications were minimal, and the reaction rate was not much greater than dapsone monotherapy. Since this is just a preliminary communication, a full report, which will include the relapse rate will be published after completion of the study.

\section{Acknowledgments}

This project received aid from Ciba-Geigy Co. Limited, and Special Programme for Research and Training in Tropical Diseases (TDR) (World Health Organization). We are also grateful for the assistance we obtained from Professor $M$ Mugambi, the Director, Kenya Medical Research Institute and Jasper K'Omollo who assisted us with data analysis.

\section{References}

I Sansarricq H. Leprosy in the world today. Lepr Rev, 1981; 52: suppl 1, 15-31.

2 Annual Report UNDP/World Bank/WHO Special Programme for Research and Training in Tropical Diseases: 7th programme report; Jan 1983-Dec 1984; (8) 1-19.

${ }^{3}$ Soutekouw PJW. Annual Report: National Leprosy and Tuberculosis Control Programme, Ministry of Health, Kenya; (1981).

${ }^{4}$ Opromolla DVA et al. A controlled clinical trial to compare the therapeutic effects of dapsone in combination with daily or once monthly rifampicin in patients with lepromatous leprosy. Int $J$ Lepr, 1981; 49: 393-7.

${ }^{5}$ Orege PA. Dapsone resistant leprosy, the Western Kenya experience. Trop Geo Med, 1985; 37: $139-42$.

${ }^{6}$ Pettit JHS, Rees RJW, Sulphone resistance in leprosy. An experimental and clinical study. Lancet, 1964; 2: 673-4.

7 Sansarricq H. Recent changes in leprosy control. Lepr Rev, 1983; Special Issue: 7-16.

${ }^{8}$ Revankar CR, Ganapati R, Nait DD, Multidrug therapy for paucibacillary leprosy, experience in Bombay. Ind J Lepr, 1985; 57: 773-9.

9 WHO Study Group. Chemotherapy of leprosy for control programmes. Technical Report Series No. 675. Who: Geneva, 1982.

${ }^{10}$ Keeler RF. Multidrug therapy for leprosy in Trinidad and Tobago: A preliminary report. Lepr Rev, 1984; 5: 391-6.

1 Rose P. Short course multidrug therapy for paucibacillary patients in Guyana. Preliminary communication. Lepr Rev, 1984; 55: 143-7. 